\title{
Clinical characteristics, treatment, and prognosis of non-B, non-C hepatocellular carcinoma: a large retrospective multicenter cohort study
}

\author{
Ryosuke Tateishi $\cdot$ Takeshi Okanoue $\cdot$ Naoto Fujiwara $\cdot$ \\ Kiwamu Okita $\cdot$ Kendo Kiyosawa $\cdot$ Masao Omata \\ Hiromitsu Kumada $\cdot$ Norio Hayashi $\cdot$ Kazuhiko Koike
}

Received: 2 April 2014/Accepted: 2 June 2014/Published online: 15 June 2014

(C) The Author(s) 2014. This article is published with open access at Springerlink.com

\begin{abstract}
Background The number of hepatocellular carcinoma (HCC) patients with non-viral etiologies is increasing in Japan. We conducted a nation-wide survey to examine the characteristics of those patients.

Methods After we assessed the trend of patients who were first diagnosed with HCC at 53 tertiary care centers in Japan from 1991 to 2010, we collected detailed data of 5326 patients with non-viral etiology. The etiologies were
\end{abstract}

For the INUYAMA NOBLESSE Study group. Members of the INUYAMA NOBLESSE Study group are listed in "Appendix".

Electronic supplementary material The online version of this article (doi:10.1007/s00535-014-0973-8) contains supplementary material, which is available to authorized users.

R. Tateishi $(\bowtie) \cdot$ N. Fujiwara · K. Koike

Department of Gastroenterology, Graduate School of Medicine,

The University of Tokyo, 7-3-1 Hongo, Bunkyo-ku,

Tokyo 113-8655, Japan

e-mail: tateishi-tky@umin.ac.jp

T. Okanoue

Saiseikai Suita Hospital, Suita, Japan

K. Okita

Ajisu Kyoritsu Hospital, Yamaguchi, Japan

K. Kiyosawa

Shironishi Hospital, Matsumoto, Japan

M. Omata

Yamanashi Prefectural Hospital Organization, Kofu, Japan

H. Kumada

Toranomon Hospital, Tokyo, Japan

N. Hayashi

Kansai Rosai Hospital, Amagasaki, Japan categorized as autoimmune hepatitis, primary biliary cirrhosis, alcoholic liver disease (ALD), non-alcoholic fatty liver disease (NAFLD), unclassified, and other. Baseline characteristics at initial diagnosis, the modality of the initial treatment, and survival status were collected via a website. Survival of the patients was assessed by the Kaplan-Meier method and Cox proportional hazard regression.

Results The proportion of patients with non-viral etiologies increased from $10.0 \%$ in 1991 to $24.1 \%$ in 2010 . Of the patients, $92 \%$ were categorized as ALD, NAFLD, or unclassified. Body mass index (BMI) was $\geq 25 \mathrm{~kg} / \mathrm{m}^{2}$ in $39 \%$. Diabetes was most prevalent in NAFLD (63\%), followed by unclassified etiology (46\%) and ALD (45\%). Approximately $80 \%$ of patients underwent radical therapy, including resection, ablation, or transarterial chemoembolization. Survival rates at $3,5,10,15$, and 20 years were $58.2,42.6,21.5,15.2$, and $15.2 \%$, respectively. Multivariate analysis revealed that patients with BMI $>22$ and $\leq 25 \mathrm{~kg} / \mathrm{m}^{2}$ showed the best prognosis versus other BMI categories, after adjusting by age, gender, tumor-related factors, and Child-Pugh score.

Conclusions Most cases of non-B, non-C HCC are related to lifestyle factors, including obesity and diabetes. Slightly overweight patients showed the best prognosis.

Keywords Hepatocellular carcinoma - Non-alcoholic fatty liver disease - Non-alcoholic steatohepatitis . Alcoholic liver disease $\cdot$ Retrospective study

\section{Introduction}

Hepatocellular carcinoma ( $\mathrm{HCC}$ ) is a typical example of an infection-associated malignancy [1]. The geographical 
distribution of the highly endemic area of HCC overlaps that of chronic hepatitis $B$ and $C$ [2]. Rigorous efforts to control horizontal transmission of hepatitis B virus (HBV) by vaccination since the mid-1980s succeeded in reducing hepatitis B-related HCC in children [3]. Screening for hepatitis $\mathrm{C}$ virus (HCV) and the ending of paid blood donations markedly reduced the incidence of transfusionassociated hepatitis [4]. In those with active chronic hepatitis $\mathrm{B}$, long-term suppression using nucleotide analogs may reduce the incidence of HBV-related HCC $[5,6]$, and the eradication of $\mathrm{HCV}$ by interferon-based therapy can reduce HCV-related HCC [7, 8]. It can reasonably be concluded that hepatitis virus-related HCC will continue to decrease in the future $[9,10]$.

While HCC is a typical example of a virus-related cancer, it is also well known to be strongly related to life style. Chronic alcoholism is a classical risk factor [11]; more recently, obesity has been recognized to strongly affect HCC development in males, versus various other malignancies [12]. There is also growing evidence suggesting that type 2 diabetes increases the incidence of HCC $[13,14]$. Due to the globally increasing proportion of the obese population over the past 30 years [15], obesityrelated $\mathrm{HCC}$ will likely continue to increase.

Unlike virus-related HCC, in which the high-risk populations and surveillance programs are well established, little is known about the characteristics of virus-unrelated HCC. To reduce the forthcoming global burden of obesity-related HCC, to clarify its clinical features is quite important. The $\underline{\text { Non-B}}$, Non-C Liver Cancer, Etiology, Prognosis and Treatment (NOBLESSE) study was conducted as a special project of the Inuyama Symposium, an assembly of 56 gastroenterology and hepatology units in university hospitals and tertiary care hospitals in Japan, to investigate the characteristics of non-B, non-C HCC patients.

\section{Patients and methods}

\section{Patients}

This retrospective study complied with the ethical guidelines for epidemiological research designed by the Japanese Ministry of Education, Culture, Sports, Science and Technology and Ministry of Health, Labour, and Welfare. The study protocol was approved by the University of Tokyo Medical Research Center Ethics Committee (approval number 3710) and the Institutional Review Board or Ethics Committee of each participating institution. Informed consent was waved because of the retrospective design. This study was registered with the University Hospital Medical Information Network (UMIN) Clinical Trial Registry (UMIN-CTR000007570).
First we collected the number of patients with $\mathrm{HCC}$ who were first diagnosed with $\mathrm{HCC}$ in the participating hospitals from 1991 to 2010 and categorized them as HBVrelated, $\mathrm{HCV}$-related, both $\mathrm{HBV}$ and $\mathrm{HCV}$-related, and non- $\mathrm{B}$, non- $\mathrm{C}$ to assess trends in the proportion of background etiologies. Next we collected detailed data of non$\mathrm{B}$, non-C HCC patients defined as negative for both hepatitis B surface antigen (HBsAg) and anti-HCV antibody. Patients who lost HBsAg before the diagnosis of HCC or who were positive for HBV DNA were excluded.

\section{Diagnosis of HCC}

The diagnosis of HCC was made by dynamic computed tomography (CT) or dynamic magnetic resonance imaging (MRI) with consideration of hyperattenuation in the arterial phase, with washout in the late phase as a definite sign of this disease [16] or pathology. In years when dynamic CT was not available, the diagnosis was also made by angiography.

\section{Data collection}

The patients were registered via a website specially designed by the investigators. The following characteristics at diagnosis were collected: age, gender, body height, body weight, etiology of background liver disease, daily alcohol consumption; comorbidities including liver cirrhosis, fatty liver by ultrasonography, hypertension, dyslipidemia, and diabetes; tumor factors including tumor size of the maximal nodule, number of tumor nodules, the presence of vascular invasion, and extrahepatic metastasis; symptoms including ascites and hepatic encephalopathy, laboratory data, including serum albumin, total bilirubin, aspartate aminotransferase (AST), alanine aminotransferase (ALT), alkaline phosphatase (ALP), gamma-glutamyl transpeptidase (GGT), platelet count, prothrombin activity, alphafetoprotein (AFP), des-gamma-carboxyprothrombin (DCP), and lens culinaris agglutinin-reactive fraction of AFP (AFP-L3); and treatment modality for the first time, including hepatic resection, liver transplantation, ablation, transarterial chemoembolization (TACE), transarterial chemotherapy, systemic chemotherapy, radiation therapy, and supportive therapy. Body mass index (BMI), ChildTurcotte-Pugh (CTP) score, and Barcelona-Clinic-LiverCancer (BCLC) stages were calculated automatically using the data obtained above.

The etiology of background liver diseases was categorized as follows: autoimmune hepatitis ( $\mathrm{AIH})$, primary biliary cirrhosis (PBC), AIH-PBC overlap syndrome, alcoholic liver disease, non-alcoholic fatty liver disease (NAFLD), Budd-Chiari syndrome, hemochromatosis, Wilson disease, and others. The diagnosis of the 
background liver disease, hypertension, dyslipidemia, and diabetes was made by the attending physician, based on the Japanese clinical guidelines for each disease. Daily alcohol consumption was calculated from forms of alcohol and frequency. Alcoholic liver disease was defined as chronic liver injury with daily alcohol consumption $\geq 80 \mathrm{~g} /$ day without another definite etiology. NAFLD was defined as a history of fatty liver or who were diagnosed with fatty liver, radiologically or pathologically, with alcohol consumption $\leq 20 \mathrm{~g} /$ day. Those with cryptogenic chronic liver disease who did not meet the criteria described above for alcoholic liver disease or NAFLD were categorized as unclassified.

Patient survival status was also registered. Status was defined as alive, dead, or lost to follow-up. Observations were censored on 31 December 2011. In diseased patients, the cause of death was categorized according to the criteria of the Liver Cancer Study Group of Japan [17], as follows: liver cancer progression, liver failure, gastrointestinal bleeding, gastro-esophageal varices rupture, rupture of liver cancer, operative death, other, and unknown.

\section{Statistical analysis}

Data are expressed as medians with 25th to 75th percentiles, unless otherwise indicated. Numbers and percentages were used for qualitative variables. Student's $t$ test was used for comparisons of two continuous variables. Differences among groups were assessed with one-way analysis of variance (ANOVA) for continuous data, and with the Chi squared test for categorical data. The Cochran-Armitage trend test was used to evaluate increasing or decreasing trends in etiology. Survival time was defined as the interval between the day of the first diagnosis and death or the last visit to the hospital until 31 December 2011. Cumulative survival curves were constructed with the Kaplan-Meier method and compared with the log-rank test. To assess the hazard ratios of various factors on overall survival, the Cox proportional hazard model was used.

Statistical analyses were performed using the ' $\mathrm{R}$ ' software (ver. 2.13.0; http://www.R-project.org). All tests were two-sided, and $p$ values $<0.05$ were considered to indicate statistical significance.

\section{Results}

Patient profiles

Of 33,782 patients who were first diagnosed with $\mathrm{HCC}$ at the 53 participating hospitals from 1991 to 2010, 5326 $(15.8 \%)$ were categorized as non-B, non-C. A marked increase in the proportion of patients categorized as non-B, non- $\mathrm{C}$ was observed ( $p<0.001$ by Cochran-Armitage test; Fig. 1). The proportion of non-B, non-C patients was $24.1 \%$ in 2010 , whereas it was only $10.0 \%$ in 1991 . The distribution of background liver diseases among non-B, non-C patients was as follows: AIH in $161(3.0 \%)$, PBC in $164(3.1 \%)$, AIH-PBC overlap syndrome in $18(0.3 \%)$, alcoholic liver disease in $1423(26.7 \%)$, NAFLD in 596 $(11.2 \%)$, Budd-Chiari Syndrome in $20(0.4 \%)$, hemochromatosis in $9(0.2 \%)$, Wilson's disease in $5(0.1 \%)$, unclassified in $2875(54.0 \%)$, and other in $53(1.0 \%)$. 'Other' included schistosomiasis japonica, suspicion of autoimmune liver diseases, and normal liver. As few patients were categorized as AIH-PBC overlap syndrome, Budd-Chiari syndrome, hemochromatosis and Wilson's disease, they were combined with 'others' in Table 1. Among non-B, non-C patients, 31 and $10 \%$ were diagnosed as HCC at the department of gastroenterology or hepatology and other department in the participating hospital, respectively. The remaining $59 \%$ were diagnosed at other hospitals and referred to the participating hospitals. Forty-one percent of patients were followed by imaging modalities before the diagnosis of HCC.

The median [interquartile range (IQR)] age in the entire cohort was 70.0 (63.0-75.0) years and approximately three-quarters were males. Patients with alcoholic liver disease were significantly younger than other etiologies $(p<0.001)$. The male to female ratio was different among the etiologies: females predominated in autoimmune liver diseases. The vast majority were non drinkers or light drinkers, except for those with alcoholic liver disease or unclassified etiology. Among those judged as unclassified, $41 \%$ were moderate drinkers.

The distribution of BMI varied across the etiologies and gender. The median BMI was the highest in those with

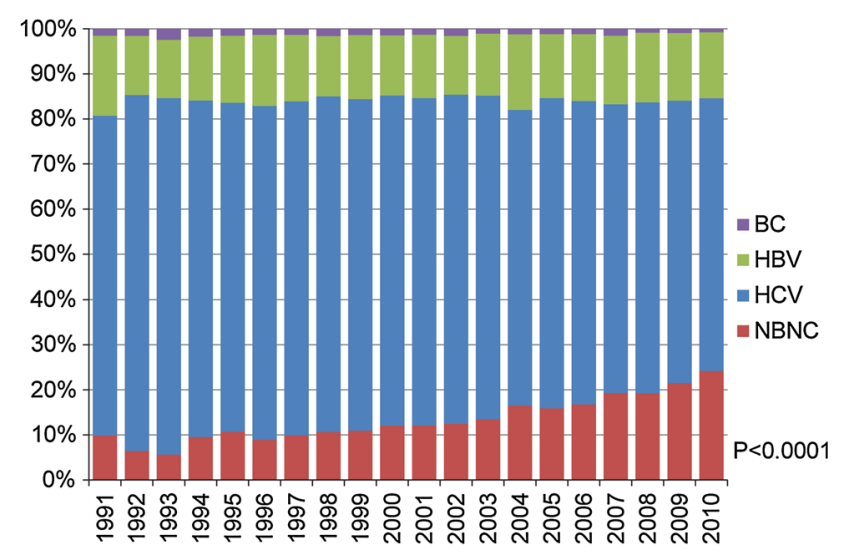

Fig. 1 Trend in background liver disease in hepatocellular carcinoma in Japan. A marked increase in the proportion of patients categorized as non-B, non- $\mathrm{C}$ in the participating hospitals was observed $(p<0.001$ by Cochran-Armitage test) 
Table 1 Baseline characteristics of the HCC patients analyzed in this study $(n=5,326)$

\begin{tabular}{|c|c|c|c|c|c|c|c|}
\hline & ALL & $\mathrm{AIH}$ & $\mathrm{PBC}$ & Alcoholic liver disease & NAFLD & Unclassified & Others \\
\hline Number of patients & 5,326 & 161 & 166 & 1,423 & 596 & 2,875 & 105 \\
\hline \multicolumn{8}{|l|}{ Age (year) } \\
\hline Median & 70.0 & 70.0 & 71.5 & 66.0 & 72.0 & 71.0 & 70.0 \\
\hline IQR & $63.0-75.0$ & $66.0-76.0$ & $66.0-77.0$ & $60.0-72.0$ & $66.0-77.0$ & $64.0-76.0$ & $58.0-76.0$ \\
\hline Male gender $[n(\%)]$ & $4,022(75.5)$ & $43(26.7)$ & $52(31.3)$ & $1,327(93.3)$ & $348(58.4)$ & $2,188(76.1)$ & $64(61.0)$ \\
\hline \multicolumn{8}{|l|}{ Alcohol consumption $(\mathrm{g} / \mathrm{day})^{\mathrm{a}}$} \\
\hline$\leq 20[n(\%)]$ & $2623(50.9)$ & $144(90.0)$ & $146(90.7)$ & & $596(100.0)$ & $1661(59.0)$ & $80(86.0)$ \\
\hline $21-79[n(\%)]$ & $1179(22.9)$ & $9(5.6)$ & $9(5.6)$ & & & $1154(41.0)$ & $7(7.5)$ \\
\hline$\geq 80[n(\%)]$ & $1351(26.2)$ & $7(3.7)$ & $6(3.7)$ & $1423(100.0)$ & & & $6(6.5)$ \\
\hline Diabetes $[n(\%)]^{\mathrm{b}}$ & $2345(46.1)$ & $48(30.6)$ & $27(17.0)$ & $621(45.2)$ & $359(62.7)$ & $1264(46.4)$ & $26(27.1)$ \\
\hline Hypertension $[n(\%)]^{\mathrm{c}}$ & $2063(42.7)$ & $51(35.4)$ & $42(26.8)$ & $493(38.0)$ & $313(55.5)$ & $1135(44.1)$ & $29(31.9)$ \\
\hline Dyslipidemia $[n(\%)]^{\mathrm{d}}$ & $720(14.6)$ & $26(17.1)$ & $12(7.6)$ & $171(12.7)$ & $125(22.9)$ & $374(14.2)$ & $12(12.6)$ \\
\hline Fatty liver $[n(\%)]^{\mathrm{e}}$ & $936(24.0)$ & $18(15.5)$ & $7(5.5)$ & $219(20.7)$ & $280(64.4)$ & $403(19.3)$ & $9(13.4)$ \\
\hline Liver Cirrhosis $[n(\%)]^{\mathrm{f}}$ & $3439(67.0)$ & $127(80.9)$ & $145(87.9)$ & $1115(80.2)$ & $368(63.4)$ & $1619(59.0)$ & $65(67.0)$ \\
\hline Anti-HBcAb positive $[n(\%)]^{g}$ & $1501(40.3)$ & $27(23.5)$ & $35(31.3)$ & $410(40.8)$ & 159 (34.6) & $837(43.0)$ & $33(40.7)$ \\
\hline \multicolumn{8}{|l|}{ ALT (U/L) } \\
\hline Median & 32 & 29 & 29 & 33 & 33 & 32 & 29 \\
\hline IQR & $22-50$ & $20-44$ & $20-41.3$ & $22-50$ & $22-51$ & $22-51$ & $20-54$ \\
\hline \multicolumn{8}{|l|}{ Platelet count $\left(\times 10^{9} / \mu \mathrm{L}\right)^{\mathrm{h}}$} \\
\hline Median & 135 & 105 & 103 & 123 & 138 & 148 & 124 \\
\hline IQR & $90-193$ & $72-166$ & $74-139$ & $84-173$ & $94-189$ & $97-205$ & $81-183$ \\
\hline \multicolumn{8}{|l|}{ Child-Pugh class ${ }^{\mathrm{i}}$} \\
\hline $\mathrm{A}[n(\%)]$ & $3500(69.0)$ & $89(57.4)$ & $83(52.9)$ & $843(62.1)$ & $439(76.5)$ & $1976(72.4)$ & $70(72.2)$ \\
\hline $\mathrm{B}[n(\%)]$ & $1231(24.3)$ & $54(34.8)$ & $57(36.3)$ & $383(28.2)$ & $120(20.9)$ & $595(21.8)$ & $22(22.7)$ \\
\hline $\mathrm{C}[n(\%)]$ & $338(6.7)$ & $12(7.7)$ & $17(10.8)$ & $131(9.7)$ & $15(2.6)$ & $158(5.8)$ & $5(5.2)$ \\
\hline \multicolumn{8}{|l|}{ Tumor characteristics } \\
\hline \multicolumn{8}{|l|}{ Maximal tumor size $(\mathrm{cm})^{\mathrm{j}}$} \\
\hline Median & 3.2 & 3.0 & 2.8 & 3.0 & 3.0 & 3.5 & 3.0 \\
\hline IQR & $2.0-6.0$ & $2.0-4.3$ & $1.7-3.5$ & $2.0-5.0$ & $2.0-5.0$ & $2.2-7.0$ & $2.0-5.1$ \\
\hline Diffuse type $[n(\%)]$ & $209(4.0)$ & $6(3.7)$ & $1(0.6)$ & $62(4.4)$ & $17(2.9)$ & $119(4.2)$ & $4(3.8)$ \\
\hline \multicolumn{8}{|l|}{ Number of nodules ${ }^{\mathrm{k}}$} \\
\hline Single $[n(\%)]$ & $2700(51.1)$ & $87(54.0)$ & $110(66.3)$ & $664(46.8)$ & $340(57.0)$ & $1443(50.8)$ & $56(53.8)$ \\
\hline $2-3[n(\%)]$ & $1368(25.9)$ & $46(28.6)$ & $40(24.1)$ & $402(28.3)$ & $156(26.2)$ & $697(24.5)$ & $27(26.0)$ \\
\hline$>3[n(\%)]$ & $1220(23.1)$ & $28(17.4)$ & $16(9.6)$ & $353(24.9)$ & $100(16.8)$ & $702(24.7)$ & $21(20.2)$ \\
\hline Vascular invasion $[n(\%)]^{1}$ & $187(3.5)$ & $3(1.9)$ & $1(0.6)$ & $52(3.7)$ & $13(2.2)$ & $116(4.1)$ & $2(1.9)$ \\
\hline Extrahepatic metastasis $[n(\%)]^{\mathrm{m}}$ & $401(7.6)$ & $8(5.0)$ & $2(1.2)$ & $114(8.0)$ & $26(4.4)$ & $244(8.6)$ & $7(6.7)$ \\
\hline \multicolumn{8}{|l|}{$\operatorname{AFP}(n g / m L)^{n}$} \\
\hline$\leq 20[n(\%)]$ & $2908(59.4)$ & $80(54.1)$ & $71(51.4)$ & $827(62.4)$ & $361(63.1)$ & $1515(58.0)$ & $54(55.7)$ \\
\hline $21-200[n(\%)]$ & $820(16.8)$ & $33(22.3)$ & $29(21.0)$ & $229(17.3)$ & $92(16.1)$ & $423(16.2)$ & $14(14.4)$ \\
\hline$>200[n(\%)]$ & $1164(23.8)$ & $35(23.6)$ & $38(27.5)$ & $270(20.4)$ & $119(20.8)$ & $673(25.8)$ & $29(29.9)$ \\
\hline \multicolumn{8}{|l|}{$\mathrm{DCP}(\mathrm{mAU} / \mathrm{mL})^{\circ}$} \\
\hline$\leq 100[n(\%)]$ & $2121(45.8)$ & $75(53.6)$ & $81(59.1)$ & $593(46.8)$ & $299(53.9)$ & $1032(42.1)$ & $41(47.7)$ \\
\hline $101-400[n(\%)]$ & $787(17.0)$ & $23(16.4)$ & $25(18.2)$ & 227 (17.9) & $95(17.1)$ & $400(16.3)$ & $17(19.8)$ \\
\hline$>400[n(\%)]$ & $1727(37.3)$ & $42(30.0)$ & $31(22.6)$ & $448(35.3)$ & $161(29.0)$ & 1017 (41.5) & $28(32.6)$ \\
\hline \multicolumn{8}{|l|}{ AFP-L3 $(\%)^{\mathrm{p}}$} \\
\hline$\leq 10[n(\%)]$ & $1765(67.7)$ & 53 (64.6) & $39(55.7)$ & 498 (69.6) & $263(73.5)$ & $881(66.1)$ & $31(66.0)$ \\
\hline $10.1-15[n(\%)]$ & $74(2.8)$ & $3(3.7)$ & $4(5.7)$ & $17(2.4)$ & $7(2.0)$ & $43(3.2)$ & $0(0)$ \\
\hline$>15[n(\%)]$ & 767 (29.4) & $26(31.7)$ & 27 (38.6) & $201(28.1)$ & 88 (24.6) & 409 (30.7) & $16(34.0)$ \\
\hline
\end{tabular}

As few patients were categorized as having the AIH-PBC overlap syndrome, Budd-Chiari syndrome, hemochromatosis or Wilson's disease, they were combined with 'others'. Data were missing in ${ }^{\mathrm{a}} 173,{ }^{\mathrm{b}} 241,{ }^{\mathrm{c}} 498,{ }^{\mathrm{d}} 388,{ }^{\mathrm{e}} 1434,{ }^{\mathrm{f}} 193,{ }^{\mathrm{g}} 1606,{ }^{\mathrm{h}} 61,{ }^{\mathrm{i}} 257,{ }^{\mathrm{j}} 42,{ }^{\mathrm{k}} 38,{ }^{\mathrm{l}} 28,{ }^{\mathrm{m}} 26,{ }^{\mathrm{n}} 434,{ }^{\mathrm{o}} 691$, and ${ }^{\mathrm{p}} 3677$ patients $A F P$ alpha-fetoprotein, AFP-L3 lens culinaris agglutinin-reactive fraction of AFP, ALT alanine aminotransferase, Anti-HBcAb anti-hepatitis B core antibody, $D C P$ des-gamma-carboxy prothrombin, $I Q R$ interquartile range 


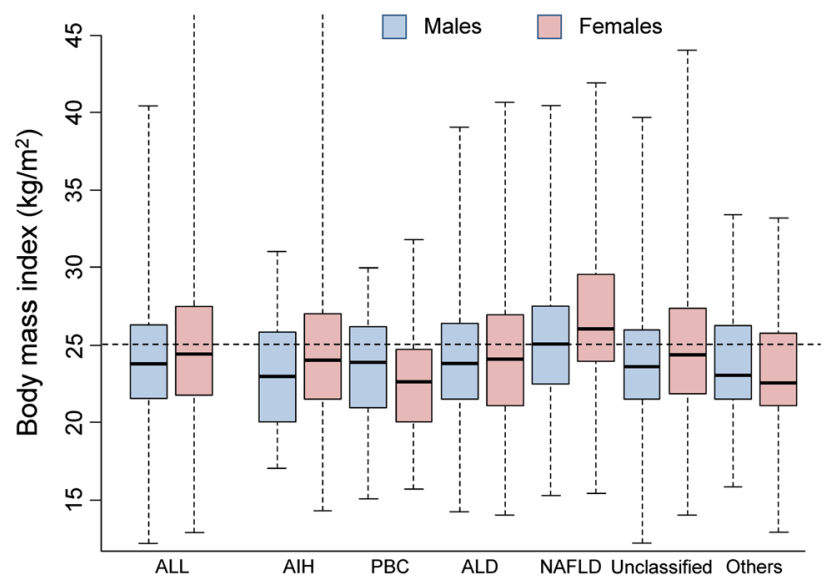

Fig. 2 Body mass index according to background liver disease. Median (25th-75th percentiles) BMI values in all categories were $23.8(21.6-26.3) \mathrm{kg} / \mathrm{m}^{2}$ in males and $24.4(21.8-27.5) \mathrm{kg} / \mathrm{m}^{2}$ in females. Box plot 'whiskers' show the minimum and maximum values; the horizontal line in each box plot shows the median, and the colored segment shows the interquartile range. $A I H$ autoimmune hepatitis, $P B C$ primary biliary cirrhosis, $A L D$ alcoholic liver disease, $N A F L D$ non-alcoholic fatty liver disease

NAFLD. Females had significantly higher BMI than males in NAFLD and those unclassified $(p=0.01$ and $<0.001$, respectively; Fig. 2).

Nearly half of the patients were complicated with diabetes (Table 1, Supplementary Fig. 1). The proportion of those with diabetes was highest in NAFLD patients. A similar trend was observed in the proportions of hypertension and dyslipidemia. The presence of fatty liver, judged by ultrasonography at the diagnosis of HCC, varied across the etiologies. The proportion was approximately $20 \%$ in alcoholic liver disease and unclassified etiology, while it was lower in autoimmune liver diseases, especially PBC. It was also suggested that fatty liver could not be detected by ultrasonography in approximately $30 \%$ at the diagnosis of HCC in NAFLD.

Approximately two-thirds of the patients were complicated with cirrhosis. The proportion of those with cirrhosis was lower in those with NAFLD and unclassified etiology compared with other etiologies $(p<0.001)$. Reflecting the proportion of cirrhosis, platelet counts were highest in those with unclassified etiology, followed by those with NAFLD.

Regarding the diagnosis process, $30.3 \%$ of the patients had their tumor pointed out for the first time in the participating department, $10.6 \%$ in another department of the same hospital, and $59.1 \%$ at other hospitals. Patients were diagnosed at more advanced stages in those with unclassified etiology; the tumor size was the largest and the proportion of patients with vascular invasion and extrahepatic metastasis was also the largest. The sensitivity of DCP was superior to that of AFP (54.2 vs. $40.6 \%$ with cutoff values of $100 \mathrm{mAU} / \mathrm{mL}$ and $20 \mathrm{ng} / \mathrm{mL}$, respectively).

Treatment and survival

Among 5058 patients in whom BCLC staging could be determined, 2533 (50.1\%), 1913 (37.8\%), $283(5.6 \%)$, and $329(6.5 \%)$ were categorized as stages A, B, C, and D, respectively (Table 2 ). The distribution of the initial treatment was as follows: resection in 1073 (20.3\%), ablation in $1060(20.0 \%)$, TACE + ablation in 470 $(8.9 \%)$, TACE in 1590 (30.1\%), transarterial chemotherapy with one-shot and continuous infusion in 99 $(1.9 \%)$, systemic therapy in $20(0.3 \%)$, radiation therapy in $20(0.4 \%)$, liver transplantation in 17 , others in 30 $(0.6 \%)$, and supportive care in $429(8.1 \%)$.

During the mean follow-up period of 2.6 years, 2225 patients died and 670 patients were lost to follow-up. The causes of death were cancer progression in 1411 (58.0\%), liver failure in 359 (14.8\%), gastrointestinal bleeding, including varices rupture, in $87(3.6 \%)$, tumor rupture in $71(2.9 \%)$, operative death in $13(0.5 \%)$, and other in 284 $(11.7 \%)$. The cause of death was unspecified in 206 $(8.5 \%)$. Median survival time $[95 \%$ confidence interval (CI)] after the initial diagnosis of HCC was 4.03 (3.82-4.20) years. Overall survival rates at 1, 3, 5, 7, 10, 15 , and 20 years were $80.1,58.2,42.6,32.2,21.5,15.2$,

Table 2 Distribution of treatments according to BCLC stage

\begin{tabular}{|c|c|c|c|c|}
\hline & A & B & $\mathrm{C}$ & $\mathrm{D}$ \\
\hline Number of patients & 2533 & 1913 & 283 & 329 \\
\hline $\begin{array}{l}\text { Hepatic resection } \\
{[n(\%)]}\end{array}$ & $616(24.3)$ & $398(20.8)$ & $30(10.6)$ & $3(0.9)$ \\
\hline Ablation $[n(\%)]$ & $887(35.0)$ & $81(4.2)$ & $4(1.4)$ & $52(15.8)$ \\
\hline $\begin{array}{l}\text { TACE + ablation } \\
{[n(\%)]}\end{array}$ & $335(13.2)$ & $116(6.1)$ & $3(1.1)$ & $4(1.2)$ \\
\hline TACE $[n(\%)]$ & $517(17.1)$ & $840(43.9)$ & 78 (27.6) & $83(25.2)$ \\
\hline $\begin{array}{l}\text { Transarterial } \\
\text { chemotherapy } \\
{[n(\%)]}\end{array}$ & $83(3.2)$ & $278(14.5)$ & $87(30.7)$ & $27(8.2)$ \\
\hline $\begin{array}{l}\text { Systemic therapy } \\
{[n(\%)]}\end{array}$ & $5(0.2)$ & $50(2.6)$ & $25(8.8)$ & $7(2.1)$ \\
\hline $\begin{array}{l}\text { Radiation therapy } \\
{[n(\%)]}\end{array}$ & $5(0.2)$ & $4(0.2)$ & $3(1.1)$ & $5(1.5)$ \\
\hline $\begin{array}{l}\text { Liver } \\
\text { transplantation } \\
{[n(\%)]}\end{array}$ & $11(0.4)$ & $6(0.3)$ & $0(0.0)$ & $0(0.0)$ \\
\hline Others $[n(\%)]$ & $12(0.5)$ & $5(0.3)$ & $2(0.7)$ & $4(1.2)$ \\
\hline $\begin{array}{l}\text { Supportive therapy } \\
{[n(\%)]}\end{array}$ & $64(2.5)$ & $135(7.1)$ & $51(18.0)$ & $144(43.8)$ \\
\hline
\end{tabular}

BCLC stage could not be determined in 268 patients

TACE transarterial chemoembolization 
Fig. 3 Overall survival.

A Overall survival of the entire patient cohort. Overall survival rates at $1,3,5,7,10,15$, and 20 years were $80.1,58.2,42.6$, $32.2,21.5,15.2$, and $15.2 \%$, respectively. B Overall survival according to BCLC stage. Survival rates at $1,3,5,7,10$, 15 , and 20 years were 94.5 , 76.4, 58.7, 44.7, 30.7, 21.9, and $21.9 \%$ in stage A, 71.1, 44.1, 29.1, 22.2, 13.0, 9.0, and $9.0 \%$ in stage B, 44.6, 18.8, 15.5, 9.3, and $9.3 \%$ in Stage C, and 48.0, 24.4, 12.3, 7.3, 3.1\%, respectively, in Stage D
A All patients

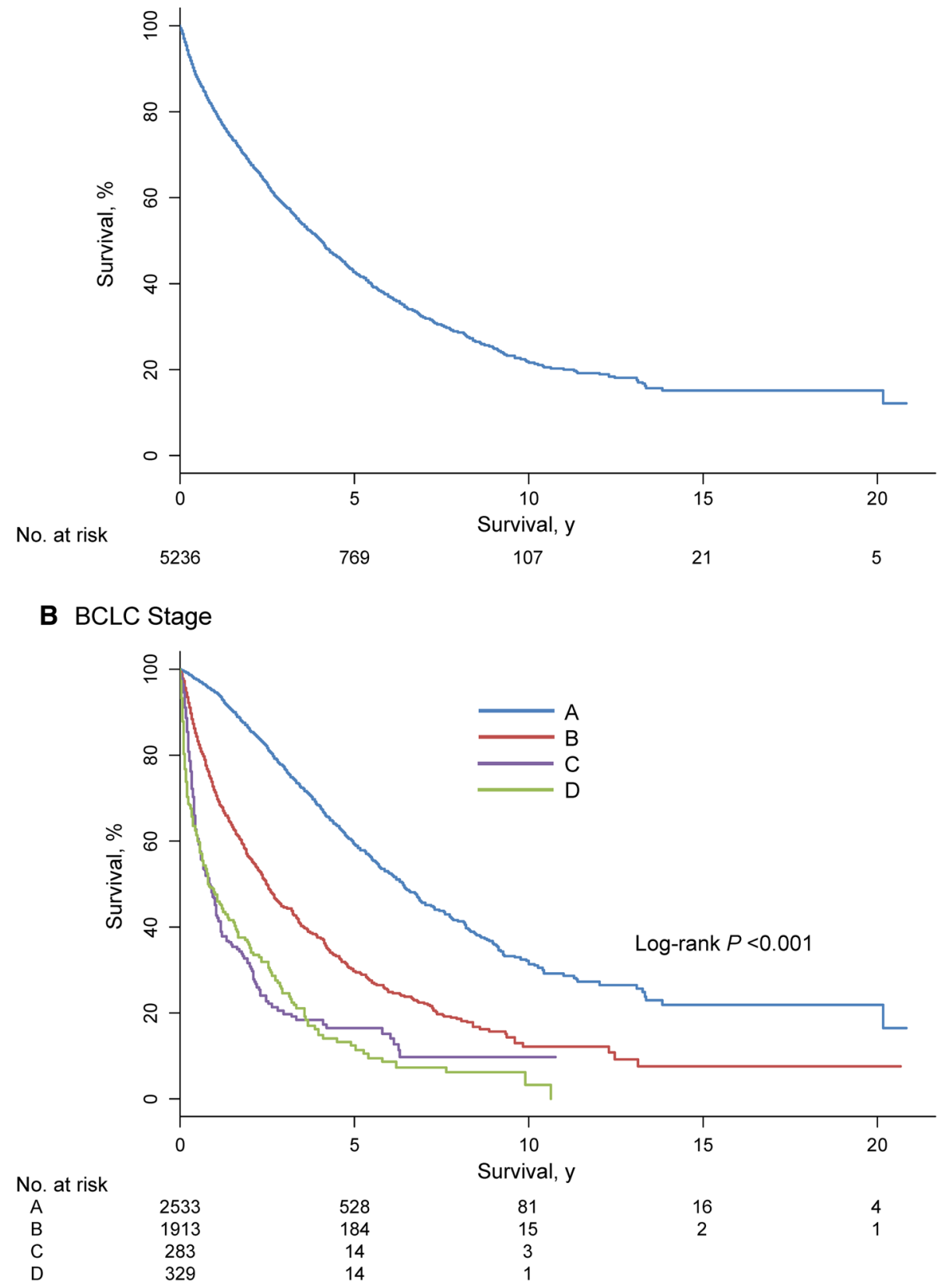

and $15.2 \%$, respectively (Fig. 3a). When stratified by BCLC stage, the median (95\% CI) survival times were 6.39 (5.96-6.85), 2.48 (2.34-2.68), 0.83 (0.61-1.03), and 0.80 (0.64-1.23) years in BCLC stages A, B, C, and D, respectively. There was a significant difference in survival among the stages (Fig. 3b, $p<0.001$ ).

Univariate Cox regression analysis revealed that the following factors were significantly related to poor survival: old age $(p<0.001)$, male gender $(p=0.003)$, alcohol consumption $\geq 80 \mathrm{~g} /$ day $\quad(p<0.001)$, BMI $(p=0.001)$, Child-Pugh score $(p<0.001)$, maximal tumor size $(p<0.001)$, number of nodules $(p<0.001)$, the presence of vascular invasion $(p<0.001)$, the presence of extrahepatic metastasis $(p<0.001)$, AFP $(p<0.001)$, DCP $(p<0.001)$, and AFP-L3 $(p<0.001)$. The presence of diabetes was indicated as a better prognosis factor, though with marginal significance (hazard ratio, 0.93; $95 \%$ CI, 0.86-1.01; $p=0.08$ ). BMI showed a V-shaped hazard distribution: those with BMIs of $22.1-25 \mathrm{~kg} / \mathrm{m}^{2}$ had the best outcomes, whereas those with higher and lower BMI showed worse prognoses. We plotted relative hazard against BMI using cubic splines. The V-shape hazard distribution was also observed in the plot (Supplementary Fig. 2). 
We performed a multivariate analysis using the variables above, except that AFP-L3 was excluded because of missing values. The results showed that age, BMI, alcohol consumption, Child-Pugh score, tumor size, number of tumor nodules, extrahepatic metastasis, AFP, and DCP were significant factors related to poor prognosis (Fig. 4). The presence of diabetes again showed no statistical significance.

\section{Discussion}

In the present study, a rapidly increasing proportion of HCC patients with non-viral etiologies was found. A similar trend was reported in a national survey by the Liver Cancer Study Group of Japan [18]. As the number of newly diagnosed HCC cases in Japan was almost at a plateau throughout the study period [19], not only the proportion,

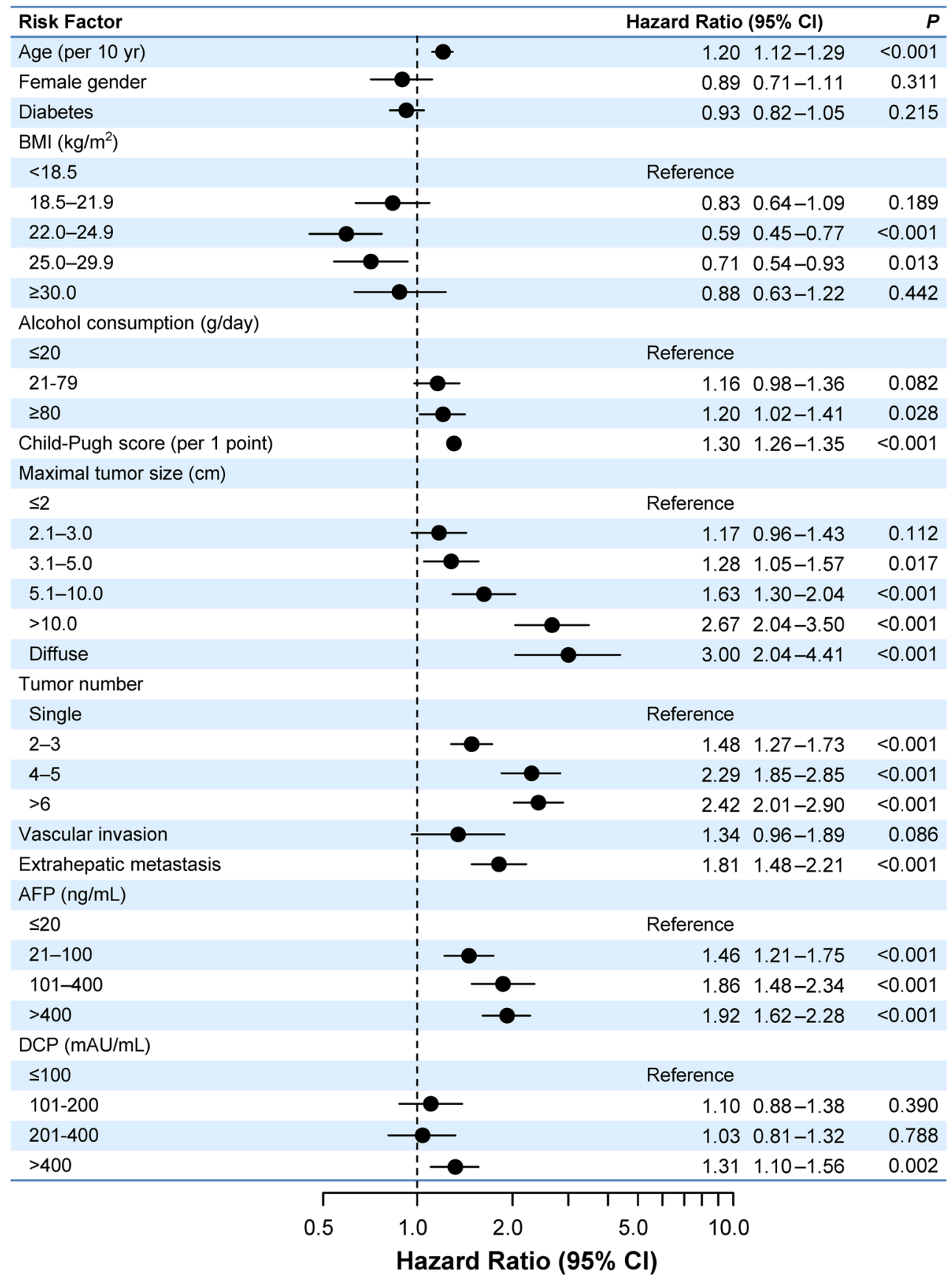

Fig. 4 Multivariate Cox proportional hazard regression analysis of survival. $A F P$ alpha-fetoprotein, $A F P-L 3$ lens culinaris agglutininreactive fraction of AFP, $D C P$ des-gamma-carboxy prothrombin $A F P$ alpha-fetoprotein, $A F P-L 3$ lens culinaris agglutinin-reactive fraction of AFP, ALT alanine aminotransferase, Anti-HBcAb anti-hepatitis B core antibody, $D C P$ des-gamma-carboxy prothrombin, $I Q R$ interquartile range 
but also the number, of patients with non-viral etiologies was increasing. As a risk factor of HCC, alcohol consumption has not increased over the last two decades in Japan according to statistics from the Ministry Labour and Welfare in Japan [20]. In contrast, the size of the obese population is increasing rapidly due to changes in the diet in Japan. The proportion of patients with diabetes has also increased in the past three decades [21]. It seems reasonable that the rapidly increasing number of HCC patients with non-viral etiologies was largely due to the rapidly increasing obese population.

Among non-viral chronic liver diseases, the natural history of AIH, PBC, and alcoholic liver disease are well known compared with that of NAFLD. In these three, HCC ordinarily arises through cirrhosis after long-lasting chronic inflammation in the liver [22-24]. Indeed, liver cirrhosis was a complication in more than $80 \%$ of those patients. In contrast, the proportion of cirrhosis was smaller and platelet counts were higher in NAFLD patients than those with $\mathrm{AIH}, \mathrm{PBC}$, and alcoholic liver disease. It has been reported that a significant proportion of patients $(41.7 \%)$ with both NAFLD and HCC are not complicated with cirrhosis [25]. That a significant proportion of patients with NAFLD or unclassified etiology were not complicated with cirrhosis suggests that to characterize a high-risk population within them would be difficult.

In this study, almost half of the patients were complicated with diabetes mellitus. According to a systematic review investigating the relationship between diabetes and $\mathrm{HCC}$, the presence of diabetes is an approximately 2.5 -fold risk of HCC [26]. Judging from the wide variation in the proportion of patients with diabetes among the etiologies, it seems that diabetes correlates more strongly with hepatocarcinogenesis in some chronic liver diseases, such as NAFLD, than others.

In this study, we defined NAFLD as a history of fatty liver and alcohol consumption of no more than $20 \mathrm{~g} /$ day. As shown in Table 1, fatty liver was not diagnosed by ultrasonography at the diagnosis of $\mathrm{HCC}$ in approximately $30 \%$ of patients with NAFLD-related HCC. Those patients would be categorized as unclassified when a history of fatty liver was not confirmed. That is, a significant proportion of those categorized as unclassified could be burn-out nonalcoholic steatohepatitis (NASH). Similarly, alcohol-related HCC could be included in unclassified patients because approximately $40 \%$ of the patients in the category were moderate drinkers. In the first place, it might be unreasonable to categorize those patients clearly, because moderate alcohol intake, obesity, and fatty liver are mutually correlated and may have a synergistic effect on hepatocarcinogenesis.

Occult infection with HBV represented by the presence of antibody to hepatitis B core antigen (anti-HBc) has been considered as a risk factor of non-B, non-C HCC defined as negative for both HBsAg and anti-HCV antibody [27, 28]. Indeed the prevalence of anti-HBc antibody was higher in this study compared to a previous report in blood donors [29]. It is also to be noted that those with anti-HBc antibody may include chronic HBV carriers with HBsAg loss before the diagnosis of HCC, who had significant risk for HCC [30].

Patients were diagnosed at less-advanced stages than we expected. This is partly because all participating hospitals were tertiary care centers. Those with terminal stages diagnosed in primary or secondary hospitals were unlikely to be referred to the participating hospitals. In addition, $41 \%$ of patients were followed by imaging modalities before the diagnosis of HCC. As a result, a large majority of patients underwent radical therapies, such as hepatic resection, ablation, or TACE, as the initial treatment.

Prognostic factors for HCC have been investigated fully in previous studies [31]. However, to our knowledge, this is the first report of the detailed relationship between BMI and survival in HCC patients. Indeed, BMI showed a $\mathrm{V}$-shaped hazard function for death. It is well known that the relationship between BMI and all-cause mortality is V-shaped, with a BMI around $22 \mathrm{~kg} / \mathrm{m}^{2}$ showing the best prognosis. However in this study, the lowest relative hazard was observed at a slightly overweight BMI. We had expected that the best BMI would be around $22 \mathrm{~kg} / \mathrm{m}^{2}$, because obesity is thought to affect hepatocarcinogenesis in this cohort and may affect recurrence after treatment. This would be because the relatively underweight patients included those with more advanced disease. However, the trend remained after adjustment for other significant factors, including those related to the tumor. Overweight patients may have some advantage versus underweight patients that we did not investigate.

The presence of diabetes did not affect survival in this study. One meta-analysis reported that the presence of diabetes increased the risk of all-cause mortality in HCC patients by 1.38 -fold (95\% CI, 1.13-1.68) [32]. It is quite reasonable that those with diabetes had additional risk for death from cardiovascular, cerebrovascular, infectious or renal diseases. Some kind of biases might exist behind the fact that the presence of diabetes did not worsen the patients' survival, which needs further investigation.

Most of the major limitations of this study relate to its retrospective design.

(1) Because the major data source was a database maintained by each participating hospital, some data were missing. Patients who were not registered in the database could not be entered into this study. However, the proportion of patients with missing data on important items, such as alcohol consumption, was less than $5 \%$; this would not affect the overall results. (2) As the amount of daily 
alcohol intake was self-reported, some patients might have underreported their alcohol intakes. Some should possibly have been categorized as having alcoholic liver disease. (3) Similarly, because the diagnosis of NAFLD was based on a past history or ultrasound examination at the diagnosis of HCC, undiagnosed burn-out NASH patients were included in those unclassified, especially when not followed in clinics or hospitals. Based on the high proportion of those with lifestyle diseases and moderate drinkers, at least a majority of those unclassified would be related to chronic alcoholism, obesity, or both.

In conclusion, the proportion of HCC patients without chronic viral hepatitis in Japan is increasing rapidly. Most had lifestyle disease-related backgrounds, especially related to obesity. Narrowing down a high-risk population would be difficult because one-third of the patients were non-cirrhotic, and obesity, fatty liver, and diabetes are prevalent in Japan.

Acknowledgments This work was supported partly by Health Sciences Research Grants of The Ministry of Health, Labour and Welfare of Japan (Research on Hepatitis). Eisai Co. supported the maintenance fee of the website for the data registration. The funders had no role in study design, data collection and analysis, decision to publish, or preparation of the manuscript.

Conflict of interest The authors declare that they have no conflict of interest.

Open Access This article is distributed under the terms of the Creative Commons Attribution Noncommercial License which permits any noncommercial use, distribution, and reproduction in any medium, provided the original author(s) and the source are credited.

\section{Appendix}

The following investigators enrolled patients in the Inuyama NOBLESSE Study: Joji Toyota, Yoshiyasu Karino (Hokkaido P.W.F.A.C Sapporo-Kosei General Hospital, Sapporo); Kazuyuki Suzuki, Hidekatsu Kuroda (Iwate Medical University, Iwate); Yoshiyuki Ueno, Hisayoshi Watanabe (Yamagata University Faculty of Medicine, Yamagata); Yutaka Aoyagi, Hirokazu Kawai (Niigata University Graduate School of Medical and Dental Science, Niigata); Eiji Tanaka, Takefumi Kimura (Shinshu University School of Medicine, Matsumoto); Kendo Kiyosawa, Hiromitsu Mori (Nagano Red Cross Hospital, Nagano); Nobuyuki Enomoto (University of Yamanashi Faculty of Medicine, Chuo); Masao Omata, Hitoshi Mochizuki (Yamanashi Central Hospital, Kofu); Satoshi Mochida, Mie Inao (Saitama Medical University, Irumagun); Kunihiko Hino, Hiromi Hoshino (Delta Clinic, Tokorozawa); Masashi Mizokami, Kazumoto Murata (Kohnodai Hospital, National Center for Global Health and Medicine, Ichikawa); Osamu Yokosuka, Fumihiko Kanai
(Chiba University Graduate School of Medicine, Chiba); Ryosuke Tateishi, Kenichiro Enooku, Koji Uchino, Masaya Sato, Shintaro Kayaki, Tatsuya Minami, Shintaro Mikami, Naoto Fujiwara (University of Tokyo Graduate School of Medicine, Tokyo); Sumio Watanabe, Kazuyoshi Kon (Juntendo University, Tokyo); Michio Imawari, Junichi Eguchi (Showa University, Tokyo); Hajime Takikawa, Masaki Mikami (Teikyo University, Tokyo); Shunji Mishiro, Masahiro Arai (Toshiba General Hospital, Tokyo); Hiromitsu Kumada, Yusuke Kawamura (Department of Hepatology, Toranomon Hospital, Kawasaki); Namiki Izumi, Takanori Hosokawa (Musashino Red-Cross Hospital, Musashino); Mitsuhiko Moriyama, Jumpei Hayashi (Nihon University School of Medicine, Tokyo); Michihiro Suzuki, Kotaro Matsunaga (Kawasaki City Tama Hospital, Kawasaki); Katsuaki Tanaka, Manabu Morimoto (Yokohama City University Medical Center, Yokohama); Takafumi Ichida, Katsuharu Hirano (Juntendo University Shizuoka Hospital, Izunokuni); Yasuhito Tanaka, Kei Fujiwara (Nagoya City University Graduate School of Medical Sciences, Nagoya); Takashi Kumada (Ogaki Municipal Hospital, Ogaki); Hisataka Moriwaki, Koji Takai (Gifu University Graduate School of Medicine, Gifu); Shuichi Kaneko, Masaaki Kitahara (Kanazawa University Graduate School of Medical Sciences, Kanazawa); Hiroshi Fukui, Masao Fujimoto (Nara Medical University, Kashihara); Yukio Osaki, Akihiro Nasu (Osaka Red Cross Hospital, Osaka); Takeshi Okanoue, Toshihide Shima (Saiseikai Suita Hospital, Suita); Toshihito Seki, Rinako Kawanura (Kansai Medical University Takii Hospital, Moriguchi); Masatoshi Kudo, Yasunori Minami (Kinki University School of Medicine, Osaka-Sayama); Tetsuo Takehara, Takayuki Yakushijin (Osaka University Graduate School of Medicine, Suita); Michio Kato, Seiji Morioka (Minami Wakayama Medical Center, Tanabe); Shuhei Nishiguchi, Hironori Tanaka (Hyogo College of Medicine, Nishinomiya); Keisuke Hino, Yasuyuki Tomiyama (Kawasaki Medical School, Kurashiki); Kazuhide Yamamoto, Kazuhiro Nouso (Okayama University Graduate School of Medicine, Dentistry and Pharmaceutical Sciences, Okayama); Kazuaki Chayama, Hiroshi Aikata (Hisoshima University Graduate School of Biomedical Sciences, Hiroshima); Isao Sakaida, Makoto Segawa (Yamaguchi University Graduate School of Medicine, Ube); Kiwamu Okita, Akira Kato (Shimonoseki Kohsei Hospital, Shimonoseki); Yoshikazu Murawaki, Naoto Maeda (Tottori University Faculty of Medicine, Yonago); Morikazu Onji, Yoichi Hiasa (Ehime University Graduate School of Medicine, Tōon); Michio Sata, Takumi Kawaguchi (Kurume University School of Medicine, Kurume); Masaru Harada, Michihiko Shibata (University of Occupational and Environmental Health, Kitakyushu); Hideyuki Nomura (Shin-Kokura Hospital, Kokura); Shotaro 
Sakisaka, Tetsuro Sohda (Fukuoka University Faculty of Medicine, Fukuoka); Masataka Seike, Koichi Honda (Oita University Faculty of Medicine, Yufu); Hiroshi Yatsuhashi, Shigemune Bekki (National Hospital Organization Nagasaki Medical Center, Omura); Kazuhiko Nakao, Naota Taura (Nagasaki University Graduate School of Biomedical Sciences, Nagasaki); Shigetoshi Fujiyama, Yoshihiro Ohuchida (Kumamoto Shinto General Hospital, Kumamoto); Yutaka Sasaki, Motohiko Tanaka (Kumamoto University Graduate School of Medicine,Kumamoto); Hirohito Tsubouchi, Tsutomu Tamai (Kagoshima University Graduate School of Medical and Dental Sciences, Kagoshima).

\section{References}

1. Parkin DM. The global health burden of infection-associated cancers in the year 2002. Int J Cancer. 2006;118(12):3030-44.

2. Jemal A, Bray F, Center MM, Ferlay J, Ward E, Forman D. Global cancer statistics. CA Cancer J Clin. 2011;61(2):69-90.

3. Chang MH, Chen CJ, Lai MS, Hsu HM, Wu TC, Kong MS, et al. Universal hepatitis B vaccination in Taiwan and the incidence of hepatocellular carcinoma in children. Taiwan Childhood Hepatoma Study Group. N Engl J Med. 1997;336(26):1855-9 [Epub 1997/06/26].

4. Yoshizawa H. Hepatocellular carcinoma associated with hepatitis $\mathrm{C}$ virus infection in Japan: projection to other countries in the foreseeable future. Oncology. 2002;62(Suppl 1):8-17.

5. Liaw YF, Sung JJ, Chow WC, Farrell G, Lee CZ, Yuen H, et al. Lamivudine for patients with chronic hepatitis B and advanced liver disease. N Engl J Med. 2004;351(15):1521-31 [Epub 2004/10/08].

6. Yokosuka O, Takaguchi K, Fujioka S, Shindo M, Chayama K, Kobashi $\mathrm{H}$, et al. Long-term use of entecavir in nucleoside-naive Japanese patients with chronic hepatitis B infection. J Hepatol. 2010;52(6):791-9.

7. Nishiguchi S, Kuroki T, Nakatani S, Morimoto H, Takeda T, Nakajima S, et al. Randomised trial of effects of interferon-alpha on incidence of hepatocellular carcinoma in chronic active hepatitis C with cirrhosis. Lancet. 1995;346(8982):1051-5 [Epub 1995/10/21].

8. Yoshida H, Shiratori Y, Moriyama M, Arakawa Y, Ide T, Sata M, et al. Interferon therapy reduces the risk for hepatocellular carcinoma: national surveillance program of cirrhotic and non-cirrhotic patients with chronic hepatitis C in Japan. IHIT Study Group. Inhibition of Hepatocarcinogenesis by Interferon Therapy. Ann Intern Med. 1999;131(3):174-81.

9. Yuen MF, Hou JL, Chutaputti A. Asia Pacific Working Party on Prevention of Hepatocellular C. Hepatocellular carcinoma in the Asia pacific region. J Gastroenterol Hepatol. 2009;24(3):346-53.

10. Tanaka H, Imai Y, Hiramatsu N, Ito Y, Imanaka K, Oshita M, et al. Declining incidence of hepatocellular carcinoma in Osaka, Japan, from 1990 to 2003. Ann Intern Med. 2008;148(11):820-6 [Epub 2008/06/04].

11. Yu MC, Tong MJ, Govindarajan S, Henderson BE. Nonviral risk factors for hepatocellular carcinoma in a low-risk population, the non-Asians of Los Angeles County, California. J Natl Cancer Inst. 1991;83(24):1820-6 [Epub 1991/12/18].

12. Calle EE, Rodriguez C, Walker-Thurmond K, Thun MJ. Overweight, obesity, and mortality from cancer in a prospectively studied cohort of U.S. adults. N Engl J Med. 2003;348(17):1625-38 [Epub 2003/04/25].

13. El-Serag HB, Tran T, Everhart JE. Diabetes increases the risk of chronic liver disease and hepatocellular carcinoma. Gastroenterology. 2004;126(2):460-8.

14. Lai MS, Hsieh MS, Chiu YH, Chen TH. Type 2 diabetes and hepatocellular carcinoma: a cohort study in high prevalence area of hepatitis virus infection. Hepatology. 2006;43(6):1295-302 [Epub 2006/05/27].

15. Finucane MM, Stevens GA, Cowan MJ, Danaei G, Lin JK, Paciorek CJ, et al. National, regional, and global trends in bodymass index since 1980: systematic analysis of health examination surveys and epidemiological studies with 960 country-years and 9.1 million participants. Lancet. 2011;377(9765):557-67.

16. The committee for revision of the Clinical Practice. Guidelines for Hepatocellular Carcinoma. Clinical Practice Guidelines for Hepatocellular Carcinoma: The Japan Society of Hepatology 2009 update. Hepatol Res. 2010;40(s1):16-47.

17. Cancer Study Group of Japan. General Rules for the Clinical and Pathological Study of Primary Liver Cancer, Second English Edition. Tokyo: Kasahara; 2003.

18. Ikai I, Arii S, Okazaki M, Okita K, Omata M, Kojiro M, et al. Report of the 17th nationwide follow-up survey of primary liver cancer in Japan. Hepatol Res. 2007;37(9):676-91.

19. Cancer Statistics in Japan. Foundation for Promotion of Cancer Research; 2012 [cited 2013 March 20th]. Available from: http:// ganjoho.jp/data/professional/statistics/backnumber/2012/cancer_ statistics_2012.pdf.

20. Higuchi S, Matsushita S, Maesato H, Osaki Y. Japan: alcohol today. Addiction. 2007;102(12):1849-62 [Epub 2007/08/08].

21. National Nutrition Survey in Japan. Ministry of Health Labour, and Welfare of Japan; 2012 [20th March]. Available from: http:// www.mhlw.go.jp/stf/houdou/2r9852000002q1st-att/ 2r9852000002q1wo.pdf.

22. Liang Y, Yang Z, Zhong R. Primary biliary cirrhosis and cancer risk: a systematic review and meta-analysis. Hepatology. 2012;56(4):1409-17.

23. Sola R, Alvarez MA, Balleste B, Montoliu S, Rivera M, Miquel $\mathrm{M}$, et al. Probability of liver cancer and survival in HCV-related or alcoholic-decompensated cirrhosis. A study of 377 patients. Liver Int. 2006;26(1):62-72.

24. Montano-Loza AJ, Carpenter HA, Czaja AJ. Predictive factors for hepatocellular carcinoma in type 1 autoimmune hepatitis. Am J Gastroenterol. 2008;103(8):1944-51 Epub 2008/06/20.

25. Ertle J, Dechene A, Sowa JP, Penndorf V, Herzer K, Kaiser G, et al. Non-alcoholic fatty liver disease progresses to hepatocellular carcinoma in the absence of apparent cirrhosis. Int J Cancer. 2011;128(10):2436-43.

26. El-Serag HB, Hampel H, Javadi F. The association between diabetes and hepatocellular carcinoma: a systematic review of epidemiologic evidence. Clin Gastroenterol Hepatol. 2006;4(3):369-80 [Epub 2006/03/11].

27. Kondo R, Nakashima O, Sata M, Imazeki F, Yokosuka O, Tanikawa K, et al. Pathological characteristics of patients who develop hepatocellular carcinoma with negative results of both serous hepatitis B surface antigen and hepatitis C virus antibody. Hepatol Res. 2013.

28. Abe H, Yoshizawa K, Kitahara T, Aizawa R, Matsuoka M, Aizawa Y. Etiology of non-B non-C hepatocellular carcinoma in the eastern district of Tokyo. J Gastroenterol. 2008;43(12):967-74.

29. Iizuka H, Ohmura K, Ishijima A, Satoh K, Tanaka T, Tsuda F, et al. Correlation between anti-HBc titers and HBV DNA in blood units without detectable HBsAg. Vox Sang. 1992;63(2):107-11.

30. Simonetti J, Bulkow L, McMahon BJ, Homan C, Snowball M, Negus S, et al. Clearance of hepatitis B surface antigen and risk 
of hepatocellular carcinoma in a cohort chronically infected with hepatitis B virus. Hepatology. 2010;51(5):1531-7.

31. Tandon P, Garcia-Tsao G. Prognostic indicators in hepatocellular carcinoma: a systematic review of 72 studies. Liver Int. 2009;29(4):502-10 [Epub 2009/01/15].
32. Yang WS, Va P, Bray F, Gao S, Gao J, Li HL, et al. The role of pre-existing diabetes mellitus on hepatocellular carcinoma occurrence and prognosis: a meta-analysis of prospective cohort studies. PLoS One. 2011;6(12):e27326. 